\title{
Zur Kenntniss der Fortpflanzung bei Arcella vulgaris Ehrb.
}

\author{
Von
}

\section{Hutschli.}

Hierzu Tafel XXV.

Seit einiger Zeit haben sich die Rhizopoden einer erhöhten Aufmerksamkeit zu erfreuen, die namentlich schon in systematischer Beziehung reichliche Früchte getragen hat, nicht ganz so jedoch in Betreff der Fortpflanzungs- und Entwicklungserscheinungen, wie dies übrigens in der Natur der Dinge begründet ist und mit dem heutigen Stande unserer Kenntnisse aufs innigste zusammenhängt. Doch sind grade diese Verhältnisse bei den in Frage stehenden niedrigsten thierischen Organismen von der allerwichtigsten principiellen Bedeutung, nicht allein für die betreffende Organismengruppe selbst, sondern für unsre gesammten Kenntnisse von dem Wesen der Zelle, dem Grundsteine aller Morphologie.

Ich beabsichtigte ursprünglich nicht, die nachstehend $\mathrm{zu}$ besprechenden Beobachtungen vereinzelt mitzutheilen, sondern im $\mathrm{Zu}$ sammenhang mit ausgedehnteren Forschungen über die Fortpflanzungserscheinungen der Infusorien. Ich werde dazu jedoch jetzt veranlasst, durch die rege Theilnahme, die den Rhizopoden von verschiedenen Seiten in letzter Zeit geschenkt wird und den Umstand, dass die Untersuchungen über die Infusorien noch eine geraume Zeit bis $\mathrm{zu}$ einem gewissen Abschluss erfordern werden.

Die neuesten Mittheilungen über die Arcellen haben R. Hertwig und E. Le s s er ${ }^{1}$ ) gemacht. Von Fortpflanzungserscheinungen haben

1) Ueber Rhizopoden und denselben nahestehende Organismen in $M$. Schultze's A. f. mikr. Anatomie Bd. X. Supplementheft. p. 97-99. 
sie einmal Encystirung und dann auch Theilung beobachtet, letztere in ähnlicher Weise, wie Clap arède unở Lachman $n^{1}$ ), die diesen Vorgang jedoch für eine Art Häutung halten zu müssen glaubten. Das Vorkommen einer wirklichen Conjugation, wie früher von Cohn angegeben wurde, glaubten sie dagegen in Abrede stellen zu müssen. Es findet sich nun aber gewiss auch Conjugation bei der Arcella vulgaris und diese Conjugation hat, wenn auch nicht immer, so doch häufig einen eigenthümlichen Forțfflanzungsprocess im Gefolge.

Hertwig und Lesser geben an, dass von den in der vermeintlichen Conjugation befindlichen Individuen stets das Fine eine tiefbraune, das Andre eine sehr helle Schale besitze, die letztere daher als eine neugebildete betrachtet werden müsse. Letzteres ist richtig, es kommen aber auch Verbindungen von zwei und auch drei Individuen vor, die sämmtlich tief braun gefärbte Schalen besitzen, wo also nur an eine Verbindung gleichwerthiger Individuen, nicht aber an eine Theilung oder Häutung gedacht werden kann.

Die erste Beobachtung über die jetzt zu beschreibende, im Gefolge der Conjugation eintretende Fortpflanzung, machte ich grade an drei mit einander conjugirten Thieren, die ich am 12. October Mittags antraf und zur weiteren fortlaufenden Beobachtung in einem kleinen Uhrgläschen in einigen Tropfen Quellwasser isolirte. Die drei in fester Verbindnng mit einanderstehenden Thiere (Fig. 1) zeigten eine gewisse Regelmässigkeit in der gegenseitigen Stellung ihrer Schalen, jedes der Thiere hatte nämlich den Schalenrand gegen die Schalenöffnung eines der andern Thiere gelehnt. Dass auch die eigentliche Leibesmasse der Arcellen in wahrer Verbindung stand, dass dieselben nicht nur etwa äusserlich aneinander hafteten, zeigte sich sehr deutlich bei der Trennung der Thiere, die nur kurze Zeit nach ihrer Isolation in dem Uhrgläschen erfolgte. Man sah vämlich dann deutlich breite Protoplasmabrücken sich noch zwischen den Schalenöffnungen der Thiere ausspannen, die schliesslich in der Mitte durchrissen und theils dem einen, theils dem andern Thiere folgten. Während der in Fig. 1 abgebildeten Vereinigung der 3 Thiere war theils wegen den ein Hinderniss bietenden Schalen, theils desshalb, weil ich die Thiere nicht ernstlich stören wollte, nicht viel von dem Verhalten ihrer Protoplasmakörper zu sehen, aus welchem Grunde auch auf Fig. 1 nur das Stellungsverhältniss der Schalen zu einan-

1) Claparède und Lachmann. Etudes sur les infusoires, T. I p. 445. 
der angegeben ist. Nachdem sich die Thiere in Laufe des Nachmittags vollständig von einander entfernt hatten, zeigte sich an ihnen weiter nichts auffallendes. Am folgenden Morgen hingegen zeigte das eine der Thiere das in Fig. 2 wiedergegebene interessante Verhalten. Der Protoplasmakörper desselben hatte sich ziemlich beträchtlich von der Schale zurückgezogen und in der ihn umgebenden Flïssigkeit wimmelten viele Vibrionen umher, ohne dass jedoch die Lebensthätigkeit des Thieres aufgehört hätte, obschon es keine Pseudopodien mehr aussendete. Auf der Rückenseite ${ }^{1}$ ) der Protoplasmascheibe lag, dieser dicht angeschmiegt, eine Doppelreihe stumpfeckiger, zellenartiger, protoplasmatischer Körper. Dieselben bildeten flache, dem Protoplasmakörper des Thieres dicht angeschmiegte Scheiben, die jedoch, wie man bei randlicher Stellung derselben erkennen konnte, mit dem Protoplasmakörper der Arcella, in dem ich nur einen Kern mit Deutlichkeit beobachtete, nicht mehr in $\mathrm{Zu}$ sammenhang standen. Im Uebrigen war die Beschaffenheit ihres Protoplasmas dem der Arcella ganz gleich. Bewegungen habe ich am Morgen des 13. an diesen Körpern noch nicht wahrgenommen. Gegen Mittag jedoch konnte man leicht erkennen, dass ein Theil der Körper sich lebhaft amöboid bewegte und zwischen dem Protoplasmakörper der Mutter und ihrer Schale herumkroch, womit natürlich auch die oben beschriebene Anordnung der fraglichen Körper verloren ging. Fernerhin liess sich jetzt auch an vielen eine pulsirende Vacuole mit aller wünschenswerthen Deutlichkeit wahrnehmen. Mit geringerer Sicherheit glaube ich auch die Theilung eines Körpers gesehen zu haben.

Um 5 Uhr Nachmittags hatte sich der Protoplasmakörper des Thieres ganz in die eine Hälfte der Schale linübergezogen (Fig. 3) und der grösste Theil der kleinen, sehr beweglichen Amöben befand sich nun in der andern Schalenhälfte. Bei genauem Zusehen ergab sich nun, dass eine derselben sich schon aussen auf der Schale dicht neben der Mündung befand. Ich setzte nun die Beobachtung anhaltend fort und konnte zu meinem grossen Vergnügen innerhalb 11/2 Stunden noch 7 andere zur Schalenöffnung herauskriechen sehen. Dieselben trieben sich eine Zeit lang auf der Schale umher, um sich von dieser zu entfernen, sobald sie einen geeigneten Stützpunkt für

1) Wenn man unter der Bauchseite die die Oeffnung tragende Schalenfläche versteht. 
ihre Weiterbewegung fanden. Um 8 Uhr Abends fand sich, dass noch eine neunte Amöbe ausgekrochen war.

Die kleinen Amöben enthalten eine contractile Vacuole und einen schwer sichtbaren, als heller Fleck sich markirenden Kern; sie bewegen sich durch Vorsehieben kurzer, sehr stumpfer Fortsätze, die sich kaum hie und da an einer Stelle einbuchten und etwas lappig werden, gewöhnlich ist nur ein einziger solcher Fortsatz in Thätigkeit.

Die kleinen Amöben selbst, die ich für die Brut der Arcella zu halten mich berechtigt glaube, konnte ich nicht in ihrer ferneren Entwicklung verfolgen, da hierzu jedenfalls die Zucht unter dem Deckgläschen unzureichend ist und die Kleinheit ihre anhaltende Verfolgung unmöglich macht.

Zunächst will ich jedoch des Schicksals der beiden andern aus der Conjugation hervorgegangenen Arcellen gedenken. - Am Abend des 13. Oct., also etwa 30 Stunden nach aufgehobener Conjugation, zeigte das zweite der Thiere an dem Rande seines Protoplasmakörpers sich grade gegenüber liegend je zwei der uns bekannten Fortpflanzungskörper, die dicht zusammenstanden. Im Gegensatz zu der raschen Entwicklung, die die Fortpflanzungskörper des erstbeschriebenen Thieres erfuhren, zeigten nun die des zweiten einen recht langsamen Entwicklungsgang, hingegen blieb in diesem Falle die Arcella selbst sehr beweglich, indem sie beständig eine mässige Zahl von Fortsätzen ausgestreckt hatte. Im Laufe des 14. nahm ich keine namhaften Veränderungen wahr; am 15. Morgens hingegen hatte sich die Zahl der Fortpflanzungskörper auf 7 erhöht (Fig. 4), von welchen jedoch noch keiner eine contractile Vakuole oder amöboide Bewegung zeigte. Drei deutliche Kerne liessen sich im Sarkodeleib des Mutterthieres wahrnehmen und das Spiel seiner nicht grade zahlreichen contractilen Vacuolen deutlich verfolgen. - Dieselben Verhältnisse zeigte das Thier noch am Morgen des 16. October. Die weitere Entwicklung dieses Thieres konnte nicht verfolgt werden, da es durch einen unglücklichen Zufall abhanden kam.

Das dritte der conjugirten Individuen starb bald nach der Trennung der Thiere $a b$, ohne dass sich an ihm eine Entwicklung von Fortpflanzungskörpern gezeigt hätte.

Ich habe nun noch eine Anzahl conjugirter Paare isolirt, über die ich jetzt noch kurz berichten will. Am 14/8. wurde ein Paar isolirt, die Thiere trennten sich wiederum sehr bald und zeigten im Laufe 
des 15. keine weitere Entwicklung. Am 16. Morgens fand ich das eine Thier in einer eigenthümlichen Verfassung, die auch von Claparède und Lachmann schon beobachtet wurde; es war nämlich ein höchst beträchtlicher Theil des Protoplasmakörpers des Thieres als eine breite scheibenförmige Masse aus der Schale herausgekrochen (Fig. 5) und hatte sogar einige, Vacuolen mit sich geführt. Als das Thier nun den 16. Abends wieder untersucht wurde, lag dicht neben ihm eine helle, dünne kreisrunde Schale (oder besser Platte) ohne weitere Structur, ohne eine Andeutung einer Oeffnung und von demselben Durchmesser wie die Schale der Arcella. Protoplasma befand sich an ihr nicht und das Protoplasma der Arcella hatte sich vollständig wieder in seine Schale zurückgezogen. Leider ging das Thier sammt seinem Gefährten bald darauf zu Grunde, so das eine weitere Entwicklung nicht festzustellen war. Es kann nun nicht wohl einem Zweifel unterliegen, dass ich hier den leider durch irgend welchen Umstand gestörten und daher unvollendet gebliebenen Vorgang der Häutung, wie ihn Claparède und Lachınann auffassen, oder der Theilung nach Hertwig und Lesser, beobachtet habe. Jedenfalls ist es von Interesse, diesen Vorgang wenigstens in seinem Beginn an einem Thier fortlaufend constatirt zu haben.

Am 16. Oct. isolirte ich wiederum ein Paar conjugirter, tiefbrauner Arcellen, die sich ebenfalls wieder sehr bald trennten. Am folgenden Morgen enthielt jedes der Thier in seiner Schale eine sehr ansehnliche Gasblase, die bei dem einen nahe die Hälfte der Schale ausfüllte. Plötzlich brach bei diesem Thier das Protoplasma in einem breiten Strom aus der Schalenöffnung hervor, ähnlich wie in dem vorhin beschriebenen Fall, die Gasblase wurde dabei mitgerissen, so dass dieselbe sich fast vollständig ausserhalb der Schale befand, im Protoplasma war sie jedoch jedenfalls nicht eingeschlossen. Am Abend desselben Tages war das Protoplasma jedes der Thiere vollständig in die Schale zurückgezogen, die Gasblase unverändert noch vorhanden.

In Bezug auf die Entwicklung von Gasblasen innerhalb der Schale, erlaube ich mir nun noch folgende Mittheilung. Ich traf sehr häufig auf Arcellen mit solchen Gasblasen, die sehr verschiedene Grösse besitzen können, häufig jedoch, wie in dem oben erwähnten Falle einen sehr ansehnlichen Durchmesser erreichen. In Bezug auf die chemische Beschaffenheit dieses Gases muss ich die Beobachtung, dass dasselbe von Kalilauge allmählich und stetig bis auf den 
letzten Rest absorbirt wird, für entscheidend halten. Es kann unter den vorliegenden Umständen kaum zweifelhaft sein, dass wir es wohl mit Kohlensäure zu thun haben, dennoch muss es sehr auffallend erscheinen, dass diese Kohlensäureblasen so lange der Absorption durch das die Arcella umspülende Wasser widerstehen sollten.

Indem wir wieder zu den beiden besprochenen, aus der Conjugation hervorgegangenen Arcellen zurückkehren, muss ich zunächst hervorheben, dass den Morgen des 18. Oct. die Gasblasen in beiden Thieren völlig verschwunden waren, ausserdem hatte sich das Protoplasma sehr vermindert oder contrahirt, die Thiere selbst bewegten sich aber noch sehr munter. Neben dem Protoplasmakörper des einen fanden sich nun aber auch wieder drei höchst deutliche Fortpflanzungskörper. Die grosse Reduction, die der Protoplasmaleib dieses Thieres schon erfahren hatte, lässt die Möglichkeit nicht vollständig von der Hand weisen, dass hier vielleicht schon junge Brut während der Nacht sich entfernt hatte, wiewohl in diesem Falle die Entwicklung eine ungemein rasche gewesen wäre. Durch ein plötzlich eintretendes Unwohlsein wurde ich nun leider verhindert, vor dem 20. Oct. wieder nach den Thieren zu sehen, wo beide abgestorben waren.

Zum Schlusse der Mittheilung des Beobachteten will ich noch kurz der eigenthümlichen Formen gedenken, die ich mehrfach sah und in Fig. 6 abgebildet habe. Die Schale dieser Arcellen hat in einer, durch die Axe der normalen Schale gehenden Ebne eine mehr oder weniger beträchtliche Krümmung erfahren, so dass die Schalenöffnung sich unterm tiefsten Punkt der concaven Einkrümmung befindet. Ich glaubte ursprünglich hier vielleicht einen Theilungszustand vor mir zu haben, indem sich ja durch Halbirung in der Symmetrieebene aus diesen Formen zwei nahezu normale Arcellen erzeugen liessen, doch hat eine mehrere Tage lang fortgesetzte Beobachtung eines derartigen Thieres nicht den geringsten Anhaltspunkt für diese Anschauung ergeben, das Thier zeigte nicht die geringste Veränderung.

Die von mir im Vorstehenden geschilderte Fortpflanzungsweise der Arcella vulgaris durch eine Amöbenbrut leidet an einer Beobachtungslücke, es ist nämlich die Frage nach der ersten Entstehung der Fortpflanzungskörper eine offene geblieben, wie wohl jedermann geneigt sein wird, dieselben auf die einfachste Weise durch einen Abschnürungs- oder Sprossungs-Process aus dem Protoplasmakörper 
der Mutter hervorgehen zu lassen. Die sichere Beobachtung dieses Punktes wäre namentlich auch desshalb von hoher Wichtigkeit, um dadurch jeden Zweifel, dass wir es hier etwa mit Parasiten zu thun hätten, die sich innerhalb der Arcellaschale entwickelten, zu bannen. Der Parasitismus hat in der Frage nach der Fortpflanzung der Protozoën schon grosse Verwirrungen angerichtet, so auf der einen Seite den ersten Anstoss zu der irrigen Meinung, dass die Infusorien Spermatozoën entwickelten und andrerseits die vermeintliche Fortpflanzung der Infusorien durch acinetenartige Embryonen, auf die $\mathrm{Häckel}$ noch neuerdings seine morphologischen Ansichten über die Infusorien basirte.

Da nun die Beobachtung über die erste Entstehung der Fortpflanzungskörper der Arcella noch nicht entschieden hat, so ist es jedenfalls von Wichtigkeit, wenn wir in der Lebensgeschichte anderer, verwandter Organismen einen ähnlichen Fortpflanzungsprocess antreffen und dies ist nach meiner Auffassung in einem ziemlichen Grade der Fall bei der Fortpflanzung der Noctiluca miliaris durch Zoosporen, wie uns die Untersuchungen von $\mathrm{Ci}$ enk o w sk $\mathrm{i}^{1}$ ) gelehrt haben. Hier sehen wir das Protoplasma des Mutterthieres hügelartige Emporstülpungen bilden, die sich ihrerseits noch mehrfach theilen und zu einer Art Scheibe auf der Oberfläche der Mutter vereinigt sind. In ganz ähnlicher Weise können und müssen wir wohl uns die Entstehung der Fortpflanzungskörper bei unserer Arcella vorstellen.

Schliesslich wird bei Noctiluca aus den Fortpflanzungskörpern eine geisselnde Zoospore, bei Arcella jedoch eine kleine recht bewegliche Amöbe, die sich vom Mutterthier entfernt. Als Hauptargumente für die Ableitung der Fortpflanzungskörper vom Protoplasma des Mutterthiers lassen sich noch anführen, die starke Reduction, die dasselbe nach der Ausbildung der Brut stets zeigt (vgl. Fig. 3), die übereinstimmende Beschaffenheit beider, und dann schliesslich, dass sich die Fortpflanzungskörper in unter dem Deckgläschen isolirten Arcellen eingestellt haben, die vorher keine Spur von ihnen zeigten. Die Conjugation aber scheint, wenn auch häufig, so doch nicht immer dem Eintreten dieser Fortpflanzungserscheinung voraus zu gehen ${ }^{2}$ ).

1) Cienkowski, Ueber Noctiluca miliaris, Sur. Archiv f. mikrosk. Anatomie, Bd. 9. p. 47.

2) Die spärlichen Beobachtungen, welche über die Fortpflanzung der 
Könnte man die jungen amöbenartigen Arcellen auf ihrem ferneren Lebenspfade verfolgen und ihre Ausbildung zu beschalten Amöben belauschen, so wäre dies gewiss der beste Weg, jeden Zweifel $\mathrm{zu}$ heben und es würde sich dabei auch die nicht unwichtige Frage lösen, wie sich die Amöbe allmählich die Arcellaschale bildet. Dies wäre jedenfalls von grossem Interesse, denn ich kann die Zweifel, die mir darüber aufgetaucht sind, ob solche Formen wie die Pseudochlamys patella $\mathrm{Cl}$ a parède und Lach mann's und die Arcella patens derselben Forscher nicht doch in den Entwicklungskreis der Arcella vulgaris gehören, nicht ganz unterdrücken.

In Betreff des Schalenbaues der Arcella vulgaris will ich mir hier am Schlusse noch eine Bemerkung erlauben. Die Schale der Arcella ist nämlich, wie man sich nur schwer am lebenden Thier, leicht jedoch an in einem gewissen Zerfall begriffenen leeren Schalen iiberzeugen kann, deutlich aus zwei Lagen aufgebaut. Die, aus hexagonalen Feldchen sich zusammensetzende hübsche Zeichnung der Arcellaschale gehört nämlich einer äusseren Schichte an, die einer inneren, der Zeichnung entbehrenden Schicht aufsitzt. Man findet nämlich leere Schalen, von welchen die äussere gefelderte Schicht theilweise abgefallen ist und überzeugt sich dabei, dass dies in der Weise geschieht, dass die einzelnen hexagonalen Feldchen sich sowohl von einander, als auch von ihrer Unterlage lösen; häufig bekommt man noch ganz vereinzelt der tieferen Schalenschicht aufsitzende hexagonale Feldchen der äusseren Schicht zur Ansicht. Es muss also die äussere gefelderte Schicht, als aus einer dichten Aneinanderreihung niederer und nach den Untersuchungen Hertwig und Lesser's hohler hexagonaier Prismen zusammengesetzt, aufgefasst werden, die durch die zerstörenden Einflüsse langer Maceration wieder aus ihrem innigen Verband gelöst werden könnnen.

Frankfurt a. M., December 1874.

eigentlichen Foraminiferen vorliegen, namentlich die von M. Schultze und Str. Wright, lassen sich mit der beschriebenen Fortpflanzung von Arcella leicht in Einklang bringen. 


\section{Erklärung der Abbildungen auf Tafel XXV.}

Fig. 1. Die in Conjugation befindlichen Thiere von Arcella vulgaris am 12. Oct. 1874. Mittags.

Fig. 2. Eines der Thiere am Morgen des 13, von der aboralen Seite gesehen.

Fig. 3. Dasselbe Thier den 13. um 5 Uhr Nachmittags, von der oralen Seite gesehen; $x$ eine schon aus der Schale gekrochene kleine Amöbe.

Fig. 4. Ein zweites Individuum aus der dreifachen Conjugation Fig. 1, von der aboralen Seite gesehen, am 15. Oct. Morgens.

Fig. 5. Ein aus der Conjugation hervorgegangenes Thier, das einen grossen Theil seines Protoplasmakörpers aus der Schale austreten hat lassen und im Begriff steht eine neue Schale zu bilden.

Fig. 6. Ein Thier mit eigenthümlicher, abnorm gebauter Schale. 


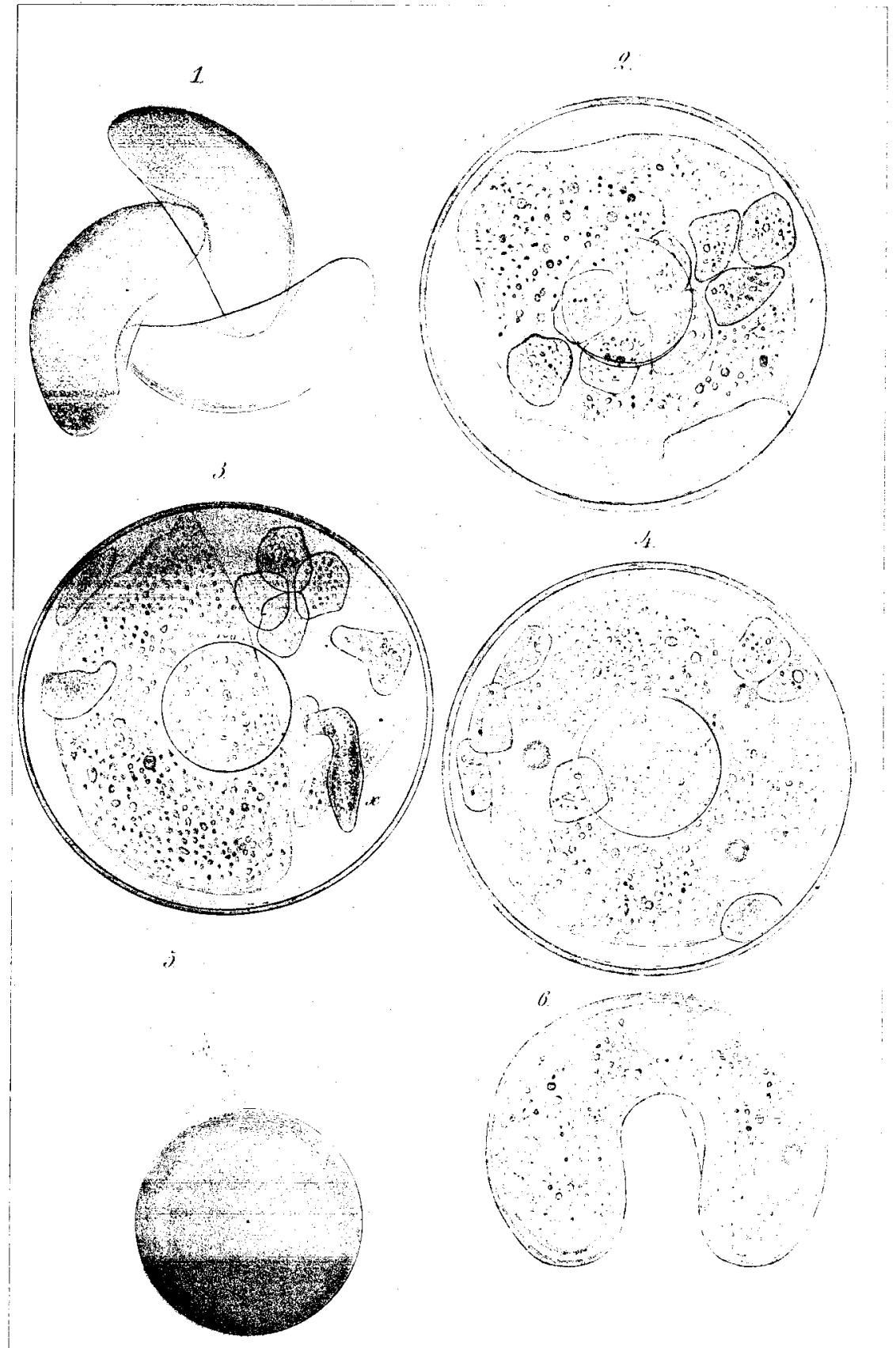

